\section{Linear N-Point Camera Pose Determination}

\author{
Long Quan and Zhongdan Lan
}

\begin{abstract}
The determination of camera position and orientation from known correspondences of $3 \mathrm{D}$ reference points and their images is known as pose estimation in computer vision and space resection in photogrammetry. It is wellknown that from three corresponding points there are at most four algebraic solutions. Less appears to be known about the cases of four and five corresponding points. In this paper, we propose a family of linear methods that yield a unique solution to 4- and 5-point pose determination for generic reference points. We first review the 3-point algebraic method. Then we present our twostep, 4-point and one-step, 5-point linear algorithms. The 5-point method can also be extended to handle more than five points. Finally, we demonstrate our methods on both simulated and real images. We show that they do not degenerate for coplanar configurations and even outperform the special linear algorithm for coplanar configurations in practice.
\end{abstract}

Index Terms-Pose estimation, space resection, 2D-3D image orientation, exterior orientation determination, perspective-n-point-problem, four points, five points.

\section{INTRODUCTION}

GIVEN a set of correspondences between 3D reference points and their images, pose estimation consists of determining the position and orientation of the calibrated camera with respect to the known reference points. The problem is called space resection in the photogrammetry community. It is one of the oldest and most important tasks in computer vision and photogrammetry. It is well-known that there are closed-form orientation solutions for three points. The first algebraic solution may be tracked back to [1841] by the photogrammetrists [1]. Many variants [2], [3], [1] of the basic 3-point algorithm have been developed ever since. Fischler and Bolles [2] give one such method that is popular in computer vision together with a RANSAC paradigm to detect outliers in the data. They also termed the problem as the perspective-3-point-problem (P3P) or, generally, PnP for any $n$ points. Church's iterative 3-point method [4] is equally widely used by photogrammetrists. Haralick et al. [1] review many old and new variants of the basic 3-point method and carefully examine their numerical stabilities due to different orders of substitution and elimination. However, additional information is required to guarantee the uniqueness of solution as there exist multiple solutions for three points. Four points generically suffice for uniqueness outside of certain critical configurations in space. All critical configurations for which multiple distinct or coinciding (unstable) solutions occur are known [5], [6]. The camera pose is ambiguous if the projection center is coplanar with any three of the four reference points or if it goes through the Euclidean horopter curve or one of its degenerate forms (the Euclidean horopter lies on a circular cylinder) uniquely determined by four given reference points.

Camera pose determination from redundant data has also been developed. However, most of them rely either on iterative methods or on applying the closed-form solutions to minimal subsets of the redundant data. Iterative methods suffer from the problems of

- L. Quan is with the CNRS-GRAVIR-INRIA, ZIRST 655, avenue de l'Europe, 38330 Montbonnot, France. E-mail: Long.Quan@inrialpes.fr.

- Zhongdan Lan is with INRS-Telecommunications, 14 Place du Commerce, Verdum, QC, Canada H3E 1H6. E-mail: zlan@inrs-telecom.uquebec.ca.

Manuscript received 8 Dec. 1997; revised 30 Mar. 1999.

Recommended for acceptance by R. Szeliski.

For information on obtaining reprints of this article, please send e-mail to: tpami@computer.org, and reference IEEECS Log Number 107783. initialization and convergence, while algebraic methods applied to subsets suffer from poor noise filtering and the difficulty of selecting the common root from the noisy data. For instance, Lowe [7] and Yuan [8] developed techniques based on Newton-Raphson iteration. Dementhon and Davis [9] presented an iterative algorithm for four points or more starting from "scaled orthographic" approximation. Horaud et al. [10] convert P4P into a special 3-line problem. Unfortunately, this conversion uses only partial information and inherits the same fourth degree polynomial equations.

Camera calibration is closely related to pose estimation, but is more general as the calibration simultaneously estimates both pose and the intrinsic parameters of the camera. Abdel-Aziz and Karara [4], Sutherland [11], and Ganapathy [12] proposed a direct linear method "DLT" for solving the 11 entries of the camera projection matrix from at least six corresponding points. The method is further improved by Faugeras and Toscani [13] using a different constraint on the projection matrix. Lenz and Tsai [14] proposed both linear and nonlinear calibration methods. Although these methods might be applied to pose determination, full calibration is a heavy over-parameterization for the pose problem, giving reduced stability and requiring more points.

Mostly in computer vision, methods for pose estimation using line segments instead of points as image features have also been developed. Dhome et al. [15] and Chen [16], developed algebraic solutions for 3-line algorithms, and Lowe [17] used the NewtonRaphson method for any number of line segments. Liu, et al. [18] combined points and line segments into the same pose estimation procedure.

Motivated by the lack of methods which directly provide a unique pose solution for the redundant data case, we develop in this paper a family of linear, unique-solution algorithms for 4-, 5-, and n-point camera pose. We therefore avoid iteration while taking advantage of data redundancy. Developing linear algorithms using redundant data for various vision tasks has always attracted attention of many researchers in computer vision [19], [20], [21], [22].

The paper is organized as follows. In Section 2, the basic 3-point closed-form solution is reviewed and discussed. Then, the linear 4point, 5-point, and N-point algorithms are successively presented in Section 3 and Section 4 . The experimental results are presented in Section 6, in which the methods developed in this paper are validated both on various simulated and real image data. The comparison is also made with the special linear algorithm for coplanar configurations. Finally, Section 7 summarizes the contributions of the paper.

\section{The Camera Pose from Three Points Revisited}

Given a calibrated camera at $C$ and $n$ correspondences between 3D reference points $\mathbf{p}_{i}$ and their images $\mathbf{u}_{i}$, each pair of correspondences $\mathbf{p}_{i} \leftrightarrow \mathbf{u}_{i}$ and $\mathbf{p}_{j} \leftrightarrow \mathbf{u}_{j}$ gives a constraint on the unknown camera-point distances $x_{i}=\left\|\mathbf{p}_{i}-\mathbf{c}\right\|$ and $x_{j}=\left\|\mathbf{p}_{j}-\mathbf{c}\right\|$ (cf. Fig. 1):

$$
d_{i j}^{2}=x_{i}^{2}+x_{j}^{2}-2 x_{i} x_{j} \cos \theta_{i j},
$$

where $d_{i j}=\left\|\mathbf{p}_{i}-\mathbf{p}_{j}\right\|$ is the known inter-point distance between the $i$ th and $j$ th reference points and $\theta_{i j}$ is the $3 \mathrm{D}$ viewing angle subtended at the camera center by the $i$ th and $j$ th points. The cosine of this viewing angle is directly computed from the coordinates of the image points and the calibration matrix $\mathbf{K}$ of the camera as 


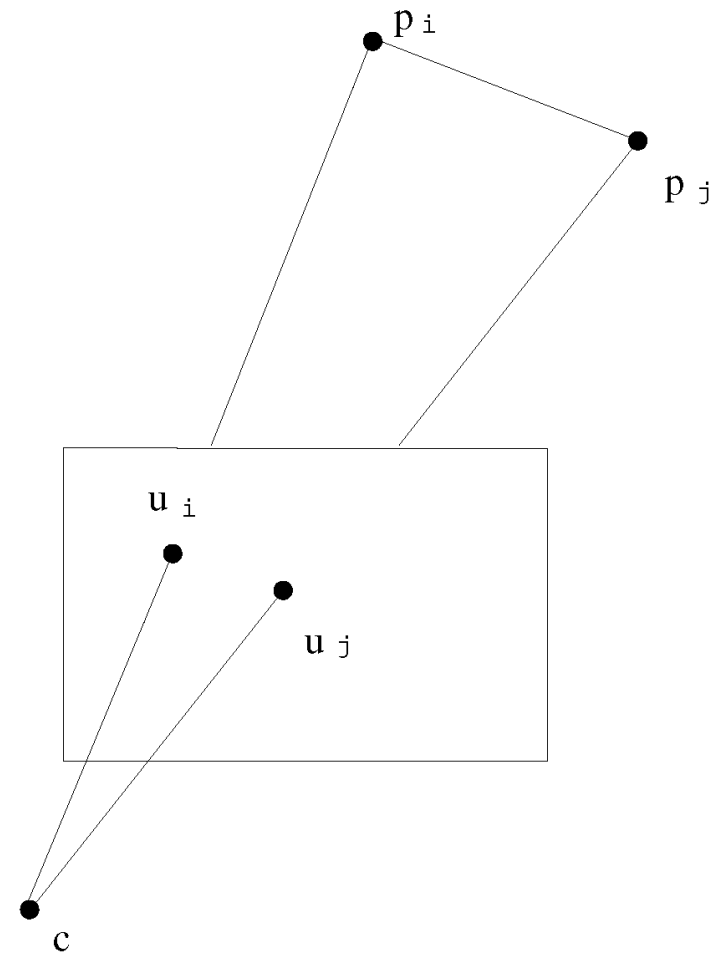

Fig. 1. The basic geometry of the camera pose determination for each pair of the correspondences $\mathbf{p}_{i} \leftrightarrow \mathbf{u}_{i}$ and $\mathbf{p}_{j} \leftrightarrow \mathbf{u}_{j}$ between the 3D reference points and their images.

$$
\cos \theta_{i j}=\frac{\mathbf{u}_{i}^{T} \mathbf{C} \mathbf{u}_{i}}{\left(\mathbf{u}_{i}^{T} \mathbf{C} \mathbf{u}_{i}\right)^{1 / 2}\left(\mathbf{u}_{j} \mathbf{C} \mathbf{u}_{j}\right)^{1 / 2}},
$$

where $\mathbf{C}=\left(\mathbf{K K}^{T}\right)^{-1}$. This formula is derived from the direction vector of the viewing line $\mathbf{K}^{-1} \mathbf{u}$. for the given image point $\mathbf{u}$.

This quadratic constraint can be rewritten as

$$
f_{i j}\left(x_{i}, x_{j}\right)=x_{i}{ }^{2}+x_{j}{ }^{2}-2 x_{i} x_{j} \cos \theta_{i j}-d_{i j}{ }^{2}=0 .
$$

For $n=3$, we obtain the following polynomial system

$$
\left\{\begin{array}{l}
f_{12}\left(x_{1}, x_{2}\right)=0 \\
f_{13}\left(x_{1}, x_{3}\right)=0 \\
f_{23}\left(x_{2}, x_{3}\right)=0
\end{array}\right.
$$

for the three unknown distances $x_{1}, x_{2}, x_{3}$. This system has a Bezout bound of $8=2 \times 2 \times 2$ solutions. However, since it has no linear terms, $x_{i} \mapsto-x_{i}$ preserves the form and the eight solutions occur in four pairs. Using classical Sylvester resultant, $x_{3}$ can be eliminated between $f_{13}\left(x_{1}, x_{3}\right)$ and $f_{23}\left(x_{2}, x_{3}\right)$ to get a polynomial $h\left(x_{1}, x_{2}\right)$. Then, further elimination of $x_{2}$ between $f_{12}\left(x_{1}, x_{2}\right)$ and $\left.h\left(x_{1}, x_{2}\right)\right)$ gives an eighth degree polynomial in $x_{1}$ with only even terms, i.e., a fourth degree polynomial in $x=x_{1}^{2}$ :

$$
g(x)=a_{5} x^{4}+a_{4} x^{3}+a_{3} x^{2}+a_{2} x+a_{1}=0 .
$$

This has at most four solutions for $x$ and can be solved in closed form. As $x_{i}$ is positive, $x_{1}=\sqrt{x}$. Then, $x_{2}$ and $x_{3}$ are uniquely determined from $x_{1}$. Note that this fourth degree polynomial is different from that derived in [2].

To obtain a unique solution, we need to add one more point. For $n=4$, an overconstrained system of six polynomials $f_{i j}\left(x_{i}, x_{j}\right)=0$ is obtained for the four unknowns $x_{1}, x_{2}, x_{3}, x_{4}$. One straightforward approach is to take subsets of three of the four points, solve the fourth degree polynomial equation for each subset of three points, and, finally, find the common
TABLE 1

Seven Possible Values of $i, j, k, l$ for Nonlinear Constraints among the Components of the Vector $t_{5}$

\begin{tabular}{|c|}
\hline$(\mathrm{i}, \mathrm{j}, \mathrm{k}, \mathrm{l})$ \\
\hline$(4,2,3,3)$ \\
\hline$(4,1,3,2)$ \\
\hline$(4,0,3,1)$ \\
\hline$(4,0,2,2)$ \\
\hline$(3,1,2,2)$ \\
\hline$(3,0,2,2)$ \\
\hline$(2,0,1,1)$ \\
\hline
\end{tabular}

solution of the subsets. This is, indeed, the common practice both in photogrammetry and computer vision. However, there are several drawbacks. First, we have to solve several fourth degree polynomials. Second, we need to find the common solution, which might be difficult due to noisy data. Finally, and probably the most important part, is that we cannot profit from the data redundancy, which should increase stability.

After having recovered camera-point distances $x_{i}$, these distances are converted into the camera-centered 3D coordinates $\tilde{\mathbf{p}}_{i}=x_{i} \mathbf{K}^{-1} \mathbf{u}_{i}$ of the reference points in space. The final step is the absolute orientation determination, a similarity transformation between two sets of $3 \mathrm{D}$ points $\tilde{\mathbf{p}}_{i} \Leftrightarrow \mathbf{p}_{i}$. The best least-square rotation is obtained in closed-form using quaternions [23], [24]. The determination of the translation and the scale follow immediately from the estimate of the rotation.

\section{The Linear 4-Point Algorithm}

The goal is to directly obtain the unique solution from a redundant polynomial equation system. Finding the common roots is equivalent to the determination of the zero-dimensional variety generated by the ideal of the polynomials. A linear polynomial in generic cases and in one of the unknowns could be algebraically obtained by successive applications of Ritt-Wu method or pseudodivision [25] of polynomials. This can effectively be done with any computer algebra system and will directly give the unique solution of the problem for general configurations of the points. However, this algebraic method is hardly useful for practical situations as the successive elimination will ultimately give complicated coefficients for the final linear polynomial which compromise the numerical stability of the solution. Instead of doing it algebraically, we to develop a numerical linear method which indeed gives the unique solution if it does exist.

For $n$ points, we have $\frac{n(n-1)}{2}$ quadratic constraints of type $f_{i j}\left(x_{i}, x_{j}\right)=0$ on the $n$ unknown distances $x_{1}, \ldots, x_{n}$, and $\frac{(n-1)(n-2)}{2}$ fourth degree polynomials of type $g(x)=0$ in one variable $x=x_{i}^{2}$.

For $n=4$, three fourth degree polynomials are given as follows: 

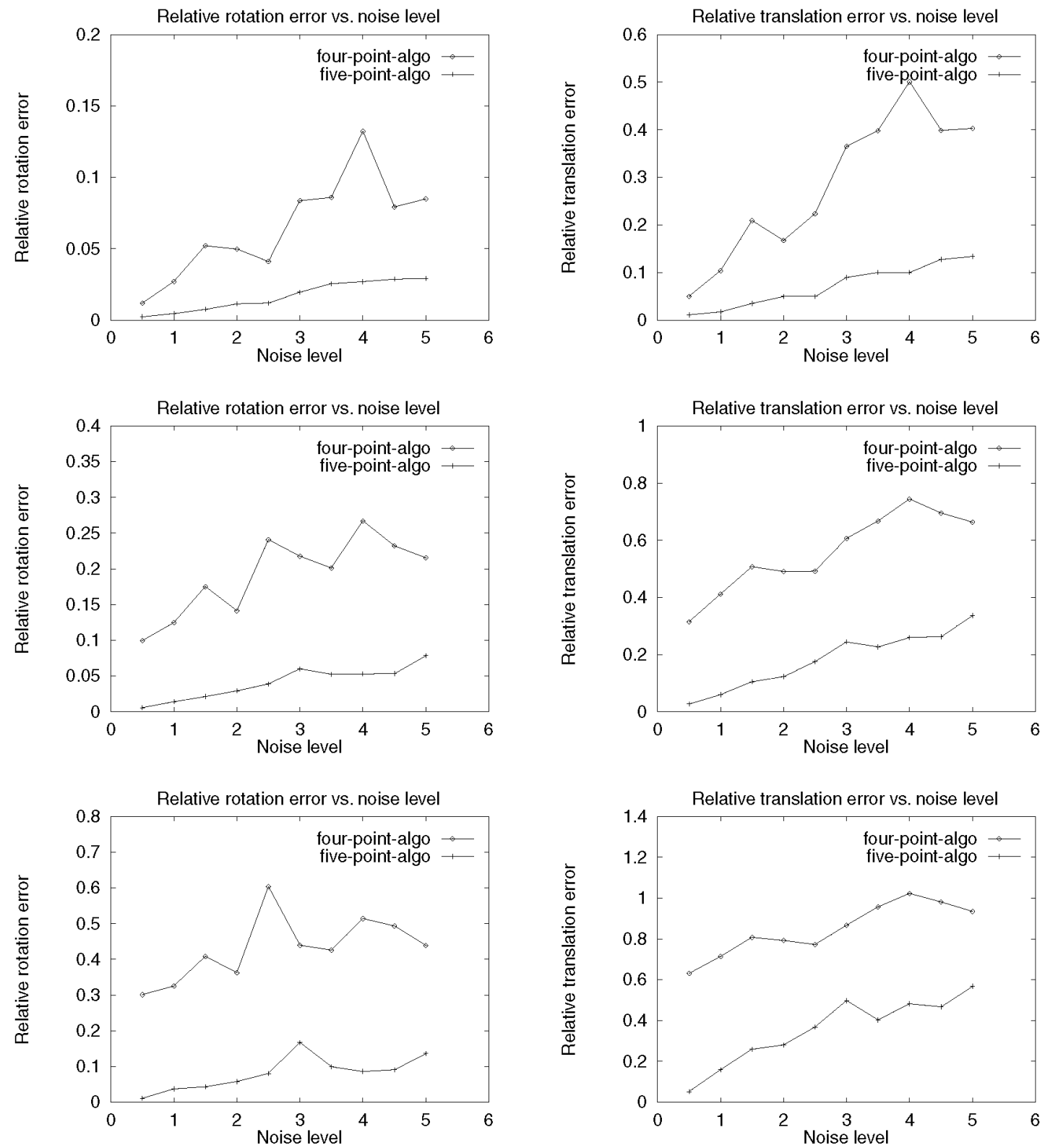

Fig. 2. The median, mean, and standard deviation of the relative errors of pose w.r.t. varying Gaussian noise levels computed by the 4-point and 5-point algorithms are shown in the first, second, and third rows. The left column is the relative rotation error and the right column is the relative translation error.

$\left\{\begin{array}{c}g(x)=a_{5} x^{4}+a_{4} x^{3}+a_{3} x^{2}+a_{2} x+a_{1}=0, \\ g^{\prime}(x)=a_{5}^{\prime} x^{4}+a_{4}^{\prime} x^{3}+a_{3}^{\prime} x^{2}+a_{2}^{\prime} x+a_{1}^{\prime}=0, \\ g(x)=a_{5} x^{4}+a_{4} x^{3}+a_{3} x^{2}+a_{2} x+a_{1}=0 .\end{array}\right.$

This can be rewritten in matrix form:

$$
\left(\begin{array}{ccccc}
a_{1} & a_{2} & a_{3} & a_{4} & a_{5} \\
a_{1}^{\prime} & a_{2}^{\prime} & a_{3}^{\prime} & a_{4}^{\prime} & a_{5}^{\prime} \\
a_{1}^{\prime \prime} & a_{2}^{\prime \prime} & a_{3}^{\prime \prime} & a_{4}^{\prime \prime} & a_{5}^{\prime \prime}
\end{array}\right)\left(\begin{array}{c}
1 \\
x \\
x^{2} \\
x^{3} \\
x^{4}
\end{array}\right)=\mathbf{A}_{3 \times 5} \mathbf{t}_{5}=0
$$

where

$$
\mathbf{t}_{5}=\left(t_{0}, t_{1}, \ldots, t_{4}\right)^{T}=\left(1, x, \ldots, x^{4}\right)^{T} .
$$

This system can be viewed as a homogeneous linear equation system in $t_{i}=x^{i}$ for $i=0, \ldots, 4$. Since the matrix $\mathbf{A}_{3 \times 5}$ has at most rank $3=\min (3,5)$, let its singular value decomposition be

$$
\mathbf{U}_{3 \times 5} \operatorname{diag}\left(\sigma_{1}, \sigma_{2}, \sigma_{3}, 0,0\right)\left(\mathbf{v}_{1}, \ldots, \mathbf{v}_{5}\right)^{T} .
$$

The null space of $\mathbf{A}_{3 \times 5}$ is spanned by the right singular vectors $\mathbf{v}_{4}$ and $\mathbf{v}_{5}$. A one-dimensional solution space for $\mathbf{t}_{5}$, parameterized by $\lambda$ and $\rho$, can be constructed as

$$
\mathbf{t}_{5}=\lambda \mathbf{v}_{4}+\rho \mathbf{v}_{5} \text { for } \lambda, \rho \in \mathbb{R} .
$$

Now consider the nonlinear constraints among the components of $\mathbf{t}_{5}$. It can be easily checked that

$$
t_{i} t_{j}=t_{k} t_{l} \text { for } i+j=k+l, 0 \leq i, j, k, l \leq 4 .
$$

Substituting $t_{i}$ from (3) in (4) gives a homogeneous quadratic equation in $\lambda$ and $\rho$ :

$$
b_{1} \lambda^{2}+b_{2} \lambda \rho+b_{3} \rho^{2}=0,
$$

where 


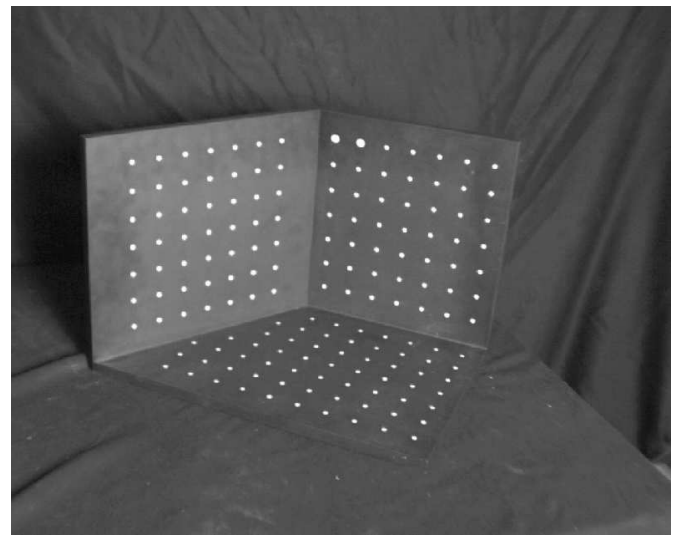

Fig. 3. The calibration pattern.

$$
\begin{aligned}
& b_{1}=\mathbf{v}_{4}^{(i)} \mathbf{v}_{4}^{(j)}-\mathbf{v}_{4}^{(k)} \mathbf{v}_{4}^{(l)}, \\
& b_{2}=\mathbf{v}_{4}^{(i)} \mathbf{v}_{5}^{(j)}+\mathbf{v}_{5}^{(i)} \mathbf{v}_{4}^{(j)}-\left(\mathbf{v}_{4}^{(k)} \mathbf{v}_{5}^{(l)}+\mathbf{v}_{5}^{(k)} \mathbf{v}_{4}^{(l)}\right), \\
& b_{3}=\mathbf{v}_{5}^{(i)} \mathbf{v}_{5}^{(j)}-\mathbf{v}_{5}^{(k)} \mathbf{v}_{5}^{(l)} .
\end{aligned}
$$

We have seven such equations for the seven different values of

$$
\{(i, j, k, l), i+j=k+l \text { and } 0 \leq i, j, k, l \leq 4\}
$$

modulo the interchanges of $i$ and $j$ or $k$ and $l$ (see Table 1).

These seven quadratic equations can be written in the following matrix form:

$$
\left(\begin{array}{ccc}
b_{1} & b_{2} & b_{3} \\
b_{1}^{\prime} & b_{2}^{\prime} & b_{3}^{\prime} \\
\vdots & \vdots & \vdots \\
b_{1}^{(6)} & b_{2}^{(6)} & b_{3}^{(6)}
\end{array}\right)\left(\begin{array}{c}
\lambda^{2} \\
\lambda \rho \\
\rho^{2}
\end{array}\right)=\mathbf{B}_{7 \times 3} \mathbf{y}_{3}=0 .
$$

Again, this overdetermined system can be viewed as linear in $\lambda^{2}$, $\lambda \rho$, and $\rho^{2}$, and solved by SVD as the right singular vector of the smallest singular value of $\mathbf{B}_{7 \times 3}$. It is clear that these seven equations are linearly, but, not algebraically independent. The constraints are the algebraic conditions for $x^{i}, i=0, \ldots, 4$, to be a geometric series.

Given the null vector $\mathbf{y}_{3}$, we solve for

$$
\lambda / \rho=y_{0} / y_{1} \text { or } \lambda / \rho=y_{1} / y_{2} .
$$

After obtaining the ratio $\lambda / \rho$, the scalars $\lambda$ and $\rho$ can be determined using one of the scalar equations of the solution (3),

$$
1=\lambda \mathbf{v}_{4}^{(0)}+\rho \mathbf{v}_{5}^{(0)} .
$$

The vector $\mathbf{t}_{5}$, is therefore, completely determined. The final $x$ is taken to be

$$
x=t_{1} / t_{0} \text { or } t_{2} / t_{1} \text { or } t_{3} / t_{2} \text { or } t_{4} / t_{3},
$$

or the average of all these values. Since $x=x_{i}^{2}$, the final depth is $x_{i}=\sqrt{x}$.

Hence, the camera pose is uniquely determined by four point correspondences provided that the four reference points together with the camera center do not lie in a critical configuration. The unique solution can be estimated by the linear 4-point algorithm.

It is important to notice that configurations of four or more coplanar points are not generically critical for this 4-point algorithm. This can be easily proven as the configuration of four coplanar points is still constrained by the six independent interpoint distances as in the noncoplanar case. It is also wellknown that the camera pose can be estimated linearly from at least four coplanar reference points [2], [26]. These two methods will be compared in Section 6.

\section{The Linear Five AND $n$-POINT AlgorithmS}

From $n=5$ on, there are sufficiently many fourth degree polynomials to directly solve $t_{i}=x^{i}$ linearly.

For the $n=5$ case, six fourth degree polynomials can be arranged into the following matrix equation:

$$
\left(\begin{array}{ccccc}
a_{1} & a_{2} & a_{3} & a_{4} & a_{5} \\
a_{1}^{\prime} & a_{2}^{\prime} & a_{3}^{\prime} & a_{4}^{\prime} & a_{5}^{\prime} \\
\vdots & \vdots & \vdots & \vdots & \vdots \\
a_{1}^{(5)} & a_{2}^{(5)} & a_{3}^{(5)} & a_{4}^{(5)} & a_{5}^{(5)}
\end{array}\right)\left(\begin{array}{c}
1 \\
x \\
x^{2} \\
x^{3} \\
x^{4}
\end{array}\right)=\mathbf{A}_{6 \times 5} \mathbf{t}_{5}=0 .
$$

Let the singular value decomposition of $\mathbf{A}_{6 \times 5}$ be $\mathbf{U}_{6 \times 6} \boldsymbol{\Sigma}_{6 \times 5} \mathbf{V}_{5 \times 5}^{T}$, the vector $\mathbf{t}_{5}$ is directly obtained as the right singular vector $\mathbf{v}_{5}$ of the smallest singular value of $\mathbf{A}_{6 \times 5}$. Then $x$ can be obtained using (5), as for the linear 4-point algorithm.

The same algorithm is also valid for any $n \geq 5$ points. We just need to SVD the matrix $\mathbf{A}$ of $\frac{(n-1)(n-2)}{2} \times 5$ to get the solution for the vector $\mathbf{t}_{5}$.

Hence, the camera pose is uniquely determined by $n \geq 5$ point correspondences provided these $n$ points, together with the camera center of the camera, do not lie in a critical configuration. The unique solution can be estimated by the linear N-point algorithm.

\section{OUtline of the Algorithms}

The linear algorithms described above can be outlined as follows:

- Data preprocessing: Compute the interpoint distances $d_{i j}=\left\|\mathbf{p}_{i}-\mathbf{p}_{j}\right\|$ and the cosines of the angular separations $\theta_{i j}$ from the image points and the camera calibration matrix $\mathbf{K}$.

- Solve for the depths of the reference points: from $(n-1)(n-2) / 2$ fourth degree polynomial equations of form $g(x)=0$, the measurement matrix $\mathbf{A} \frac{(n-1)(n-2)}{2} \times 5$ can be formed, then SVD is applied to $\mathbf{A}$ either in two steps for four points or in one step for five or more points. The square of the depth $x$ is obtained from (5), then the depth is set to $x_{i}=\sqrt{x}$.

- Absolute orientation: The recovered depths of the reference points are converted into a camera rotation and translation using the absolute orientation method briefly described in Section 2.

\section{Experimental Results}

We first demonstrate the accuracy and stability of the linear 4- and 5-point algorithms, both on simulated and real image data. Then, the linear 4-point method is also compared with the special linear algorithm for four coplanar points.

\subsection{The Linear 4- and 5-Point Algorithm}

We first simulate a number of images in which 4- or 5-point correspondences are given. The coordinates of four or five reference points in 3D are randomly generated within a cube of width 200 by a uniform random number generator. The orientation Euler angles of the camera are also positioned randomly. The reference points are projected onto an image plane using the camera pose and internal parameters. The focal length is set to 1,500 and the principal point to $(256,256)$. One hundred camera poses are generated and 100 sets of points are generated for each 

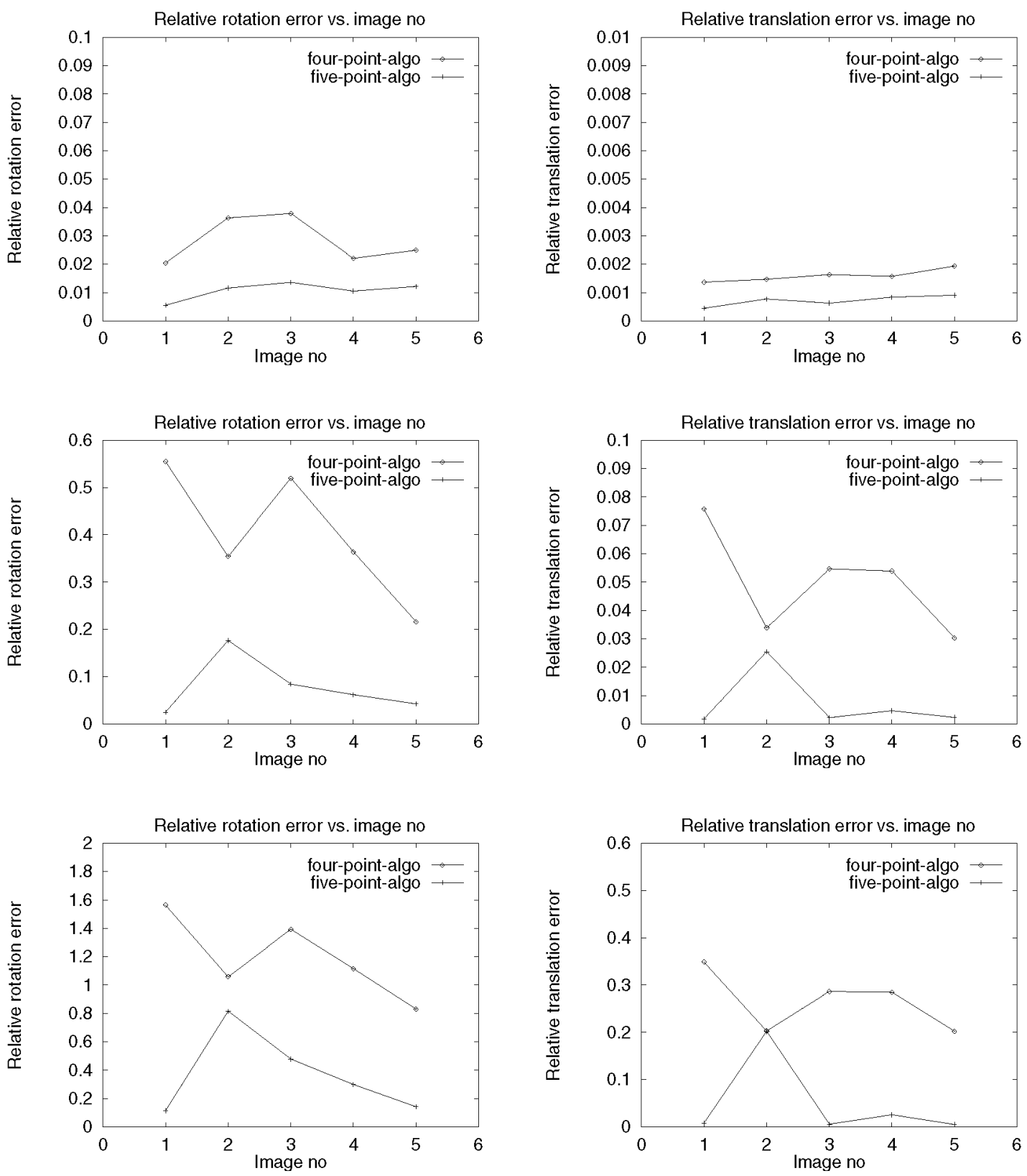

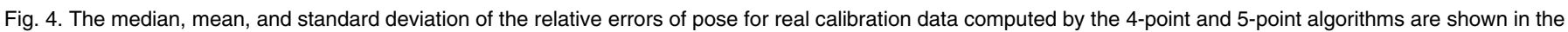
first, second, and third rows. The left column is the relative rotation error and the right column is the relative translation error.

camera pose. The positions of the image points are perturbed by Gaussian noises.

The relative error of the estimated translation $\mathbf{t}_{i}$ w.r.t. the true $\mathbf{t}$ is measured by 2|| $\mathbf{t}_{i}-\mathbf{t}|| /\left(\left\|\mathbf{t}_{i}\right\|+\| \mathbf{t}||\right)$. The relative error of the estimated rotation $\mathbf{R}_{i}$ w.r.t. the true $\mathbf{R}$ is measured by the sum of the absolute values of the three Euler angles of the relative rotation $\mathbf{R}_{i} \mathbf{R}^{T}$.

The median, mean, and standard deviation of the relative errors w.r.t. varying noise levels are illustrated in Fig. 2. We observe that both 4- and 5-point algorithms yield very graceful degradation with increasing noises and are, therefore, very stable. The 5-point algorithm slightly outperforms the 4-point one as its data redundancy is higher.

The algorithms are also demonstrated on real image data from the calibration pattern illustrated in Fig. 3. Five images of the pattern are taken and about 160 target points are accurately extracted from each image [27]. A complete camera calibration is carried out for each image the camera pose from the calibration matrix serves as ground truth. One hundred sets of four and five points are drawn randomly from the extracted targets. The same procedure as in the simulated data case for the pose estimation and result analysis carried out. The median, the mean, and the standard deviation of the relative error are illustrated in Fig. 4 in which we see very good stabilities of the algorithms.

\subsection{The Linear 4-point Versus the Special Linear Algorithm for Coplanar Points}

The coplanar configurations are of particular interest since there exists an exact linear algorithm for the unique pose solution that we have already mentioned in Section 3. At first, we use a similar simulation set-up as in Section 6.1 to generate sets of coplanar points. The relative errors w.r.t. varying noise levels are illustrated in Fig. 5. Then, we use a real quasi-coplanar data set to compare the two methods. This real data set contains 28 points making a cross shape, but the two bars of the cross are not exactly sitting on 

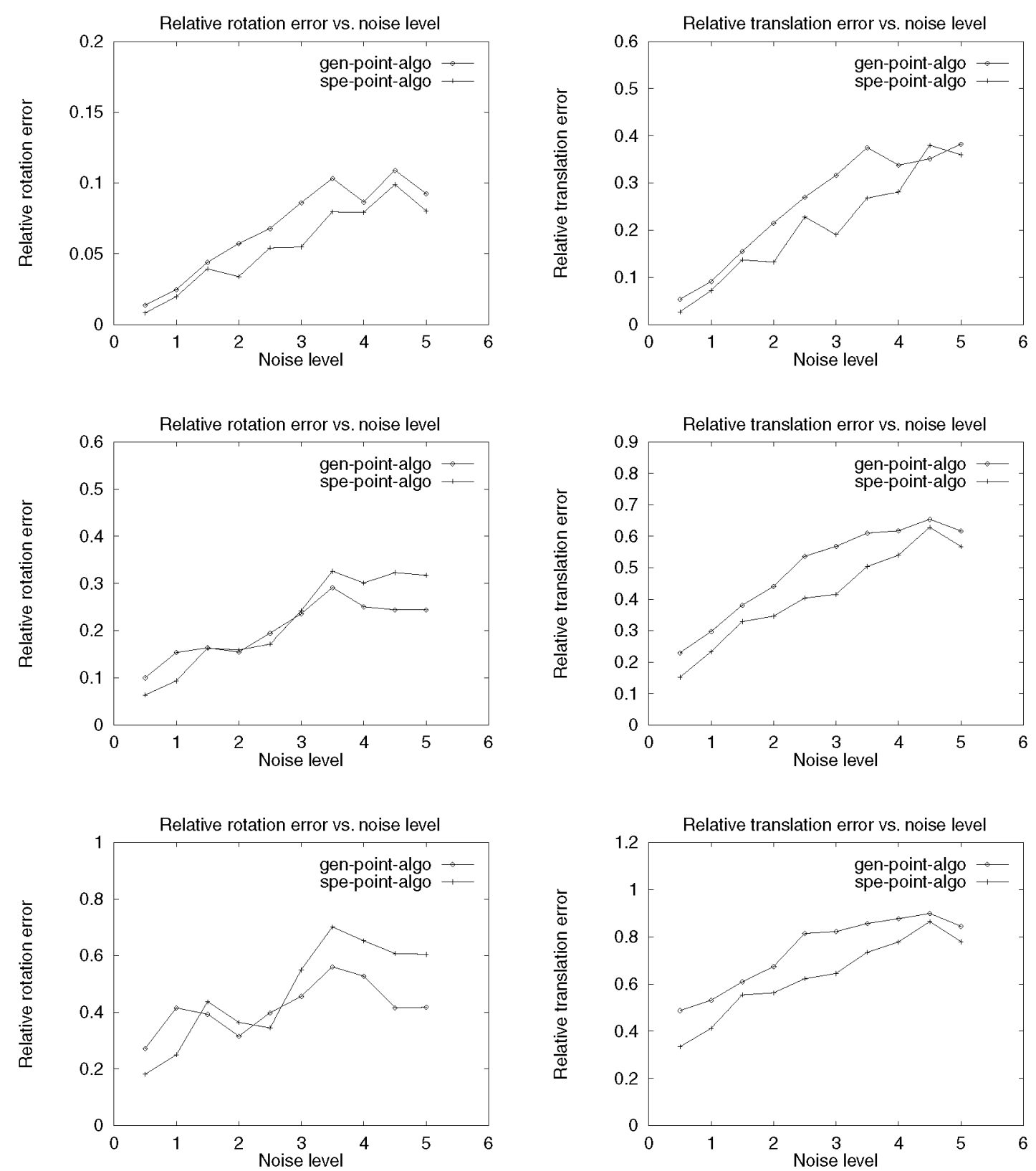

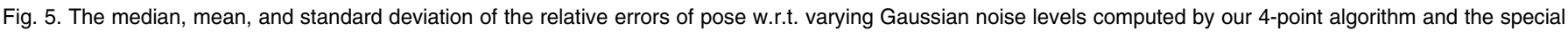

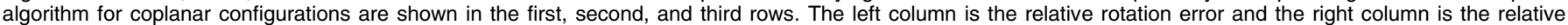
translation error.

the same plane; the noncoplanarity is about 5 percent. The results obtained from different poses and different sets of points of this quasi-coplanar configuration are shown in Table 2.

We observe that our 4-point method performs almost as well as the special linear four point algorithm for configurations of coplanar points. And, the 4-point method clearly outperforms the special linear one for quasicoplanar configurations. Some large relative errors are also observed in Table 2 for the special linear algorithm. This corresponds to configurations of the four points which are degenerate for it but not for our method. For instance, when three of the four points are aligned, the special linear algorithm fails.

\section{Discussion}

We have presented a family of linear algorithms for N-point pose determination. For four points, a two-step linear algorithm was developed and, for $n \geq 5$ points, a simple one-step algorithm was developed. These linear algorithms give a unique solution whenever the reference points are not sitting on one of the the known critical surfaces. The main advantages of this family of linear algorithms can be summarized as follows: The unicity of solution is guaranteed, they do not degenerate for coplanar configurations, and even outperform the special linear algorithm for quasi-coplanar points. Finally, the methodology developed in this paper can easily be applied to other problems in vision with overconstrained systems of polynomial equations.

\section{ACKNOWLEDGMENTS}

This work was partly supported by the European LTR project CUMULI. The authors would like to thank B. Triggs and R. Mohr for many fruitful discussions. Zhongdan Lan is also grateful for the support of INRS-telecommunication under CRSNG. 
TABLE 2

The Relative Error in Percentage of the Computed Depth by the Special Linear Algorithm (SL) and the linear 4-point (L) for each different camera position of the quasicoplanar real image data.

\begin{tabular}{|c|c|c|c|c|c|c|c|c|c|c|c|}
\hline \#test & 1 & 2 & 3 & 4 & 5 & 6 & 7 & 8 & 9 & 10 & median \\
\hline SL & 22.3 & 4.0 & 8.3 & 0.07 & 1.9 & 17.5 & 0.09 & 0.5 & 2.9 & 0.3 & 2.4 \\
\hline L & 0.4 & 0.02 & 0.3 & 0.01 & 0.1 & 0.09 & 0.03 & 0.1 & 0.4 & 0.4 & 0.4 \\
\hline SL & 0.8 & 2.7 & 0.9 & 0.3 & 7.9 & 17.6 & 0.27 & 2.3 & 9.9 & 1.2 & 1.75 \\
\hline L & 0.08 & 0.008 & 12.1 & 0.04 & 3 & 0.3 & 0.01 & 0.02 & 0.06 & 0.2 & 0.07 \\
\hline SL & 7.3 & 12 & 2.0 & 12.4 & 11.6 & 1.0 & 40.6 & 3.2 & 0.4 & 23.8 & 9.45 \\
\hline L & 0.03 & 0.08 & 0.1 & 0.3 & 0.03 & 0.04 & 2.0 & 1.2 & 0.005 & 1.0 & 0.09 \\
\hline SL & 2.1 & 33.6 & 0.05 & 8.7 & 0.3 & 6.9 & 1.2 & 5.8 & 0.06 & 1.9 & 2 \\
\hline L & 0.02 & 0.1 & 0.1 & 0.04 & 0.03 & 0.2 & 0.07 & 0.1 & 0.7 & 0.01 & 0.085 \\
\hline SL & 1.6 & 16.1 & 8.5 & 3.8 & 1.7 & 8.9 & 6.6 & 0.02 & 0.3 & 0.3 & 2.75 \\
\hline L & 0.01 & 0.01 & 0.06 & 0.05 & 0.2 & 0.1 & 0.07 & 0.01 & 0.05 & 0.02 & 0.05 \\
\hline SL & 4.7 & 5.7 & 11.5 & 8.4 & 3.0 & 4.3 & 4.0 & 0.1 & 28.7 & 1.0 & 4.5 \\
\hline L & 0.09 & 0.6 & 0.1 & 0.05 & 0.07 & 0.6 & 0.8 & 0.02 & 0.2 & 0.05 & 0.095 \\
\hline
\end{tabular}

\section{REFERENCES}

[1] R.M. Haralick, C. Lee, K. Ottenberg, and M. Nölle, “Analysis and Solutions of the Three Point Perspective Pose Estimation Problem," Proc. IEEE Conf. Computer Vision and Pattern Recognition, pp. 592-598, Maui, Hawaii, 1991.

[2] M.A. Fischler and R.C. Bolles, "Random Sample Consensus: A Paradigm for Model Fitting with Applications to Image Analysis and Automated Cartography," Graphics and Image Processing, vol. 24, no. 6, pp. 381-395, June 1981.

[3] W. Förstner, "Reliability Analysis of Parameter Estimation in Linear Models with Applications to Mensuration Problems in Computer Vision," Computer Vision, Graphics and Image Processing, vol. 40, pp. 273-310 1987.

[4] Manual of Photogrammetry, fourth ed., C.C. Slama, ed. Falls Church, Va., Am. Soc. Photogrammetry and Remote Sensing, 1980.

[5] E.H. Thompson, "Space Resection: Failure Cases," Photogrammetric Record, vol. X, no. 27, pp. 201-204, 1966.

[6] B.P. Wrobel, "Minimum Solutions for Orientation," Proc. IEEE Workshop Calibration and Orientation Cameras in Computer Vision, Washington, D.C., Aug. 1992.

[7] D. Lowe, "Fitting Parameterized Three-Dimensional Models to Images," IEEE Trans. Pattern Analysis and Machine Intelligence, vol. 13, no. 5, pp. 441450, May 1991.

[8] J.S.C. Yuan, "A General Phogrammetric Solution for the Determining Object Position and Orientation," IEEE Trans. Robotics and Automation, vol. 5, no. 2, pp. 129-142, Apr. 1989.

[9] D. Dementhon and L.S. Davis, "Model-Based Object Pose in 25 Lines of Code," Int'l J. Computer Vision, vol. 15, no. 1, pp. 123-141, 1995.

[10] R. Horaud, B. Conio, O. Leboulleux, and B. Lacolle, "An Analytic Solution for the Perspective 4-Point Problem," Computer Vision, Graphics, and Image Processing, vol. 47, pp. 33-44, 1989.

[11] I.E. Sutherland, "Three-Dimensional Input by Tablet," Proc. IEEE, vol. 62, pp. 453-461, 1974.

[12] S. Ganapathy, "Decomposition of Transformation Matrices for Robot Vision," Proc. IEEE Conf. Robotics and Automation, pp. 130-139, 1984.

[13] O.D. Faugeras and G. Toscani, "Camera Calibration for 3D Computer Vision," Proc. Int'l Workshop Machine Vision and Machine Intelligence, Tokyo, 1987.

[14] R.K. Lenz and R.Y. Tsai “Techniques for Calibration of the Scale Factor and Image Center for High Accuracy 3D Machine Vision Metrology," Proc. IEEE Int'l Conf. Robotics and Automation, pp. 68-75, Raleigh, N.C., 1987.

[15] M. Dhome, M. Richetin, J.T. Lapresté, and G. Rives, "Determination of the Attitude of 3D Objects from Single Perspective View," IEEE Trans. Pattern Analysis and Machine Intelligence, vol. 11, no. 12, pp. 1,265-1,278, Dec. 1989.

[16] H.H. Chen, "Pose Determination from Line-to-Plane Correspondence: Existence Condition and Closed-Form Solutions," Proc. Third Int'l Conf. Computer Vision, pp. 374-378, Osaka, Japan, 1990.

[17] D.G. Lowe, Perceptual Organization and Visual Recognition. Norwell, Mass. Kluwer Academic Publishers, 1885.
[18] Y. Liu, T.S. Huang, and O.D. Faugeras, “ Determination of Camera Location from 2D to 3D Line and Point," IEEE Trans. Pattern Analysis and Machine Intelligence, vol. 12, no. 1, pp. 28-37, Jan. 1990.

[19] H.C. Longuet-Higgins, "A Computer Program for Reconstructing a Scene from Two Projections," Nature, vol. 293, pp. 133-135, Sept. 1981.

[20] M. Spetsakis and J. Aloimonos, "Structure from Motion Using Line Correspondences," Int'l J. Computer Vision, vol. 4, pp. 171-183, 1990.

[21] Y. Liu and T.S. Huang, "A Linear Algorithm for Motion Estimation Using Straight Line Correspondences," Computer Vision, Graphics, and Image Processing, vol. 44, no. 1, pp. 35-57, Oct. 1988.

[22] J. Philip, "A Noniterative Algorithm for Determining All Essential Matrices Corresponding to Five Point Pairs," Photogrammetric Record, vol. 15, no. 88, pp. 589-599, Oct. 1996.

[23] B.K.P. Horn, "Closed Form Solution of Absolute Orientation Using Unit Quaternions," J. Optical Soc. Am., vol. 5, no. 7, pp. 1,127-1,135, 1987.

[24] O. Faugeras and M. Hebert, "The Representation, Recognition, and Locating of 3D Objects," Int'l J. Robotics Research, vol. 5, pp. 27-52, 1986.

[25] B.W. Char, K.O. Geddes, G.H. Gonnet, B.L. Leong, M.B. Monagan, and S.M. Watt, Maple V. Springer-Verlag, 1992.

[26] P.R. Wolf, Elements of Photogrammetry. New York: McGraw-Hill, 1974.

[27] P. Brand, "Reconstruction Tridimensionnelle d'une Scène à Partir d'une Caméra en Mouvement de l'Influence de la Précision," Thèses de Doctorat, Université Claude Bernard, Lyon I, Oct. 1995. 